\title{
HAUSDORFF HYPERCUBES WHICH DO NOT CONTAIN ARCLESS CONTINUA
}

\author{
MICHEL SMITH
}

\begin{abstract}
A Hausdorff arc is a compact connected Hausdorff space with exactly two noncut points. The finite product of a Hausdorff arc is called a Hausdorff hypercube. Suppose that $X$ is a Hausdorff arc which is first countable at none of its points and $n$ is a positive integer. We show that every nondegenerate subcontinuum of $X^{n}$ contains a Hausdorff arc. Thus $X^{n}$ contains no nondegenerate hereditarily indecomposable continuum.
\end{abstract}

During our study of nonmetric hereditarily indecomposable continua we have discovered that if $X$ is a Hausdorff arc which is not first countable at any of its points, then the Hausdorff hypercube, $X^{n}$ for $n$ a positive integer, does not contain a nondegenerate hereditarily indecomposable continuum ${ }^{1}$. We later discovered that the argument generalizes to a proof that, if $X$ is a Hausdorff arc which is not first countable at any of its points, then every nondegenerate subcontinuum of $X^{n}$ for $n$ a positive integer contains a Hausdorff arc. A Hausdorff arc is defined as a compact connected Hausdorff space with exactly two noncut points. However, Grispolakis and Tymchatyn [GT] have shown that each Hausdorff continuum of $\operatorname{dim} \geq 2$ contains an indecomposable continuum.

Let us introduce some notation. Suppose that $X$ is a Hausdorff arc with endpoints $x_{0}$ and $x_{1}$. Then there is an order relation $<$ on $X$ in the order from $x_{0}$ to $x_{1}$ and the order topology induces the topology of $X$. Suppose $a \in X$ and $b \in X$. Then let

$$
[a, b]=\{x \mid a \leq x \leq b\}, \quad(a, b)=\{x \mid a<x<b\} .
$$

Suppose $x \in X$. Then we say that $x$ is a type I point if some countable infinite sequence of points converges to $x$ and $x$ is a type II point if no countable infinite sequence of points converges to $x$.

THEOREM 1. There exists a Hausdorff arc $X$ which is first countable at none of its points.

Proof. Consider $\omega_{1}$, the first uncountable ordinal. Let $X$ be the collection of all functions from $\omega_{1}$ into $[0,1]$. Define an order relation on $X$ as follows: If $f \in X$ and $g \in X$ let $f<_{X} g$ if and only if $f(\alpha)<g(\alpha)$ with respect to the order on $[0,1]$, where $\alpha$ is the first ordinal $\lambda<\omega_{1}$ such that $f(\lambda) \neq g(\lambda)$. The order $<_{X}$ is a generalized lexicographic ordering. It is not difficult to prove that $X$ with the order topology induced by $<_{X}$ is compact, connected, and linearly ordered; hence $X$ is a Hausdorff arc. Suppose now that $X$ is first countable at some point $x=\{x(\alpha)\}_{\alpha<\omega_{1}}$. Let us assume that $x$ is not an endpoint of $X$, only a slight

Received by the editors August 20, 1984 and, in revised form, November 2, 1984.

1980 Mathematics Subject Classification. Primary 54F20.

Key words and phrases. Hausdorff arc, nonmetric arc, indecomposable continua.

${ }^{1}$ Presented at the Topology Seminar, Banach Center, Warsaw, Poland, April-June 1984 
modification of the following argument is needed in case $x$ is an endpoint. Then there is a countable sequence $\left\{\left(a_{i}, b_{i}\right)\right\}_{i=1}^{\infty}$ which forms a basis at $x, a_{i}<x<b_{i}$. Let $\alpha_{i}$ be the first ordinal $\lambda$ so that $a_{i}(\lambda) \neq x(\lambda)$ and let $\beta_{i}$ be the first ordinal $\lambda$ so that $b_{i}(\lambda) \neq x(\lambda)$. So we have $a_{i}\left(\alpha_{i}\right)<x\left(\alpha_{i}\right)$ and $x\left(\beta_{i}\right)<b_{i}\left(\beta_{i}\right)$. Thus since $\left\{\lambda \mid \lambda=\alpha_{i}\right.$ or $\lambda=\beta_{i}$ for some positive integer $\left.i\right\}$ is countable there is an ordinal $\delta$ so that $\alpha_{i}<\delta$ and $\beta_{i}<\delta$ for all positive integers $i$. Either $x(\lambda) \neq 0$ for uncountably many $\lambda$ or $x(\lambda) \neq 1$ for uncountably many $\lambda$. Let us suppose without loss of generality that $x(\lambda) \neq 0$ for uncountably many $\lambda$. Let $\delta^{\prime}$ be such that $\delta<\delta^{\prime}$ and $x\left(\delta^{\prime}\right) \neq 0$. Let $y \in X$ be defined as follows:

$$
\begin{array}{ll}
y(\lambda)=x(\lambda) & \text { if } \lambda<\delta^{\prime} \\
y(\lambda)=0 & \text { if } \delta^{\prime} \leq \lambda .
\end{array}
$$

Then $y \neq x$ and, since $y(\lambda)=x(\lambda)$ for $\lambda<\delta^{\prime}, y \in\left(a_{i}, b_{i}\right)$ for all $i$. Thus $\left\{\left(a_{i}, b_{i}\right)\right\}_{i=1}^{\infty}$ is not a basis at $x$, and we have a contradiction. Thus if $x \in X$ then $X$ is not first countable at $x$.

LEMMA 2.1. Suppose $X$ is a Hausdorff arc and $A_{1}, A_{2}, \ldots$ is an infinite sequence of points in $X^{n}$. Then some subsequence has a sequential limit point.

PROOF. If $a_{1}, a_{2}, \ldots$ is an infinite subsequence of $X$ then there is either an infinite increasing subsequence of $\left\{a_{i}\right\}_{i=1}^{\infty}$ or an infinite decreasing subsequence of $\left\{a_{i}\right\}_{i=1}^{\infty}$. So, in either case, there is an infinite subsequence of $\left\{a_{i}\right\}_{i=1}^{\infty}$ with sequential limit point. Let $A_{i}=\left(a_{i}^{1}, a_{i}^{2}, \ldots, a_{i}^{n}\right)$. Let us also assume without loss of generality that $\left\{a_{i}^{j}\right\}_{i=1}^{\infty}$ is infinite for $j=1$. Then some infirite subsequence $\left\{a_{n_{i}}^{1}\right\}_{i=1}^{\infty}$ has a sequential limit point. So some infinite subsequence of $\left\{a_{n_{i}}^{2}\right\}_{i=1}^{\infty}$ has a sequential limit. And by induction we can get a sequence of integers $\left\{l_{i}\right\}_{i=1}^{\infty}$ so that $\left\{a_{l_{i}}^{1}\right\}_{i=1}^{\infty}$ is infinite and has a sequential limit and $\left\{a_{l_{i}}^{j}\right\}_{i=1}^{\infty}$ has a sequential limit $j=1,2,3, \ldots, n$. Therefore, $\left\{A_{l_{i}}\right\}_{i=1}^{\infty}$ is a subseqence of $\left\{A_{i}\right\}_{i=1}^{\infty}$ with a sequential limit.

LeMmA 2.2. Suppose $X$ is a Hausdorff arc. Then if $M_{1}, M_{2}, \ldots$ is a countable sequence of closed subsets of $X$ and $M$ is the limiting set of the sequence, and $M_{i} \cap M=\emptyset$ for all $i$, then each point of $M$ is of type $\mathrm{I}$.

ProOF. Suppose $x \in M$ and $x$ is a type II point. If $i$ is a positive integer then there exists an open set $\left(r_{i}, s_{i}\right)$ so that $r_{i}<x<s_{i}$ and $\left(r_{i}, s_{i}\right) \cap M_{i}=\emptyset$. Since $x$ is a type II point and $r_{i}<x$ for all $i$ then there exists a point $r \in X$ so that $r_{i}<r<x$ for all $i$. Similarly, there is a point $s \in X$ so that $x<s<s_{i}$ for all $i$. Thus, $(r, s)$ is an open set containing $x$ but no point of $\bigcup_{i=1}^{\infty} M_{i}$. This is a contradiction.

LEMMA 2.3. If $X$ is a Hausdorff arc which is first countable at none of its points then the set of type I points of $x$ is dense in $X$ and the set of type II points is dense in $X$.

PROOF. It is sufficient to prove the theorem to show that every such arc contains a type I point and a type II point. Suppose then that $X=[a, b]$ is a Hausdorff arc which is first countable at none of its points and $X$ is ordered from $a$ to $b$ with respect to $<$. Let $\left\{x_{i}\right\}_{i=1}^{\infty}$ be a countably infinite sequence of points of $X$. Then by Lemma 2.1. some subsequence has a sequential limit point which would be a type I point. 
Now let us establish that $X$ contains a type II point. Let $G$ be the set to which $g$ belongs if and only if $g=[r, s]$ for some $r<s$ in $X$. Let $G$ be well ordered. By transfinite induction a subcollection $J$ of $G$ may be constructed so that

(1) the first element of $G$ belongs to $J$,

(2) if $j \in J$ and $L(j)=\{g \in J \mid g<j\}$ then $j$ is the first element of $G$ which is a proper subset of every element of $L(j)$ and which has no endpoint in common with any element of $L(j)$, and

(3) $J$ is maximal with respect to properties (1) and (2) above.

Then, since $J$ is maximal, $\bigcap\{j \mid j \in J\}$ is a degenerate set $\{z\}$. We claim that $z$ is a type II point. Since $X$ is not first countable at $z$ and $z$ is the endpoint of no element of $J$ then no countable set is cofinal in $J$. Suppose that $z$ is not a type II point. Then let $x_{1}, x_{2}, \ldots$ be a sequence of points distinct from $z$ which has sequential limit $z$. For each integer $n$ there exist an element $j_{n} \in J$ so that $x_{n} \notin j_{n}$. Let $I=\bigcap_{n=1}^{\infty} j_{n}$, then $x_{n} \notin I$ for all positive integers $n$. But $z$ is a limit point of $\left\{x_{i}\right\}_{i=1}^{\infty}$ so $z$ must be an endpoint of $I$. But $X$ is not first countable so no countable set is cofinal in $J$ and there is a first element $j^{\prime}$ in $J$ which follows every element of the set $\left\{j_{n}\right\}_{n=1}^{\infty}$. By the definitions of $J$ and $I, j^{\prime} \subset I$. Let $j^{\prime \prime}$ be the first element of $J$ that follows $j^{\prime}$. Then $z \in j^{\prime \prime}$ and $j^{\prime \prime}$ intersects neither endpoint of $j^{\prime}$, hence neither endpoint of $I$. This is a contradiction. Therefore $z$ is a type II point.

Let $\pi_{i}: X^{n} \rightarrow X$ be the natural projection map, so if $x=\left(x^{1}, x^{2}, \ldots, x^{n}\right)$ then $\pi_{i}(x)=x^{i}$.

LEMMA 2.4. Suppose $X$ is an arc, $n$ is a positive integer, and $M$ is a nondegenerate subcontinuum of $X^{n}$ so that if $K$ is a subcontinuum of $M, k$ is an integer, $1 \leq k \leq n$, and $r<s$ in $X$, then the number of components of $K \cap$ $\left(X^{k-1} \times[r, s] \times X^{n-k-1}\right)$ that intersect both $K \cap\left(X^{k-1} \times\{r\} \times X^{n-k-1}\right)$ and $K \cap\left(X^{k-1} \times\{s\} \times X^{n-k-1}\right)$ is finite. Then $M$ contains an arc.

PROOF. We prove the lemma by induction on $n$ the number of factors of $X^{n}$. Thus let $m$ be an integer so that if $n<m$ and $M$ is a subcontinuum of $X^{n}$ which satisfies the hypothesis then $M$ contains an arc. Suppose $M$ is a subcontinuum of $X^{n}$ which satisfies the hypothesis of the lemma but which contains no arc. For some $k, 1 \leq k \leq m, \pi_{k}(M)$ is nondegenerate. Without loss of generality we assume that $k=1$. Let $r$ and $s$ be such that $[r, s] \subset \pi_{1}(M)$.

Suppose $K$ is a subcontinuum of $M$ and $u<v$. Then let $K_{[u, v]}=K \cap([u, v] \times$ $\left.X^{m-1}\right)$ and $K_{u}=K \cap\left(\{u\} \times X^{m-1}\right)$. Therefore, by the induction hypothesis, if $K$ is a subcontinuum of $M$ then $K_{u}$ must be totally disconnected. (Otherwise, $K_{u}$ contains a copy of a nondegenerate subcontinuum of $X^{m-1}$ which satisfies the hypothesis of the lemma.)

Let $I$ be a subcontinuum of $M$ irreducible from $M_{r}$ to $M_{s}$. Let $p \in I-M_{r} \cup M_{s}$. Let $\Gamma$ be an index set such that if $r<a<\pi_{1}(p)<b<s$ then $a=a_{\gamma}$ and $b=b_{\gamma}$ for some $\gamma \in \Gamma$. Let $r<a_{\gamma}<p<b_{\gamma}<s$ and let $L_{\gamma}$ be the component of $I_{\left[a_{\gamma}, b_{\gamma}\right]}$ that contains $p$. If $H \subset I$ let $\operatorname{Int}_{I}(H)$ denote the interior of $H$ with respect to $I$ and let $\mathrm{Bd}_{I}(H)$ denote the boundary of $H$ with respect to $I$.

Claim a. Suppose $q \in L_{\gamma}$ for some $\gamma, a_{\gamma}<\pi_{1}(q)<b_{\gamma}$. Then $q \in \operatorname{Int}_{I}\left(L_{\gamma}\right)$.

PROOF. Suppose not. Then there are infinitely many components of $I_{\left[a_{\gamma}, b_{\gamma}\right]}$. Let $\left\{L_{i}\right\}_{i \in A}$, where $A$ is some infinite index set, be an infinite sequence of such 
components so that $q$ is a limit point of $\bigcup_{i \in A} L_{i}$. But then there exists a number $t$ such that either $\pi_{1}(q)<t$ and $I_{\left[t, b_{\gamma}\right]}$ has infinitely many components intersecting both $I_{t}$ and $I_{b_{l}}$ or $t<\pi_{1}(q)$ and $I_{\left[a_{\gamma}, t\right]}$ has infinitely many components intersecting both $I_{a_{\gamma}}$ and $I_{t}$. This contradicts the fact that $M$ satisfies the hypothesis of the lemma. This proves Claim a.

For $\gamma \in L$ let $J_{\gamma} \subset I$ be a continuum irreducible from $I_{r}$ to $L_{\gamma}$ and let $K_{\gamma} \subset I$ be a continuum irreducible from $I_{s}$ to $L_{\gamma}$.

Claim b. $J_{\gamma} \cap L_{\gamma} \subset I_{a_{\gamma}} \cup I_{b_{\gamma}}$ and $K_{\gamma} \cap L_{\gamma} \subset I_{a_{\gamma}} \cup I_{b_{\gamma}}$.

PROOF. Suppose $J_{\gamma} \cap L_{\gamma} \not \subset I_{a_{\gamma}} \cup I_{b_{\gamma}}$ and $q \in J_{\gamma} \cap L_{\gamma}-I_{a_{\gamma}} \cup I_{b_{\gamma}}$. So $a_{\gamma}<\pi_{1} q<b_{\gamma}$ and, by Claim a, $q \in \operatorname{Int}_{I} L_{\gamma}$. But since $J_{\gamma}$ is irreducible from $I_{r}$ to $L_{\gamma}$, every point of $J_{\gamma} \cap L_{\gamma}$ is a limit point of $J_{\gamma}-J_{\gamma} \cap L_{\gamma}$, which is a contradiction. Similarly, $K_{\gamma} \cap L_{\gamma} \subset I_{a_{\gamma}} \cup I_{b_{\gamma}}$.

Therefore, we have $I=J_{\gamma} \cup L_{\gamma} \cup K_{\gamma}$, and since $p \notin J_{\gamma} \cup K_{\gamma}$ we also have $J_{\gamma} \cap K_{\gamma}=\emptyset$.

Claim c. $J_{\gamma}$ is unique and $K_{\gamma}$ is unique.

PROOF. Suppose that $J_{\gamma}$ is not unique and that $J$ is a subcontinuum of $I$ irreducible from $I_{r}$ to $L_{\gamma}$ which is distinct from $J_{\gamma}$. Suppose that $J$ contains a point $z$ not in $J_{\gamma}$. Then $J$ contains a point $z^{\prime}$ not in $L_{\gamma} \cup J_{\gamma}$. The continuum $J$ must also satisfy Claim b, so $J \cap K_{\gamma}=\emptyset$. So $z^{\prime} \notin L_{\gamma} \cup J_{\gamma} \cup K_{\gamma}$. But then $J_{\gamma} \cup L_{\gamma} \cup K_{\gamma}$ is a proper subcontinuum of $I$ which intersects $I_{r}$ and $I_{s}$, which is a contradiction. Similarly, $J_{\gamma}$ cannot contain a point not in $J$. A similar argument proves that $K_{\gamma}$ is unique. This proves Claim c.

Claim d. If $\gamma_{1}$ and $\gamma_{2}$ are such that $a_{\gamma_{1}}<a_{\gamma_{2}}<\pi_{1}(p)<b_{\gamma_{2}}<b_{\gamma_{1}}$, then $J_{\gamma_{1}} \subset \operatorname{Int}_{I}\left(J_{\gamma_{2}}\right)$ and $K_{\gamma_{1}} \subset \operatorname{Int}_{I}\left(K_{\gamma_{2}}\right)$.

PROOF. Since $J_{\gamma_{1}}$ and $J_{\gamma_{2}}$ are unique we have $J_{\gamma_{1}} \subset J_{\gamma_{2}}$. Since $I=J_{\gamma_{i}} \cup L_{\gamma_{i}} \cup$ $K_{\gamma_{i}}$ then, by Claim b, $\operatorname{Bd}_{I}\left(J_{\gamma_{i}}\right)=J_{\gamma_{i}} \cap L_{\gamma_{i}} \subset\left(I_{a_{\gamma_{i}}} \cup I_{b_{\gamma_{i}}}\right)$. Also by definition of $L_{\gamma}$ we have $L_{\gamma_{2}} \subset L_{\gamma_{1}}$. So $L_{\gamma_{2}} \cap L_{\gamma_{1}}=L_{\gamma_{2}}$. So

$$
\begin{aligned}
\left(\operatorname{Bd}_{I} J_{\gamma_{2}}\right) \cap J_{\gamma_{1}} & =\left(J_{\gamma_{2}} \cap L_{\gamma_{2}}\right) \cap J_{\gamma_{1}} \\
& =\left(J_{\gamma_{2}} \cap L_{\gamma_{2}} \cap L_{\gamma_{1}} \cap J_{\gamma_{1}}\right) \\
& \subset\left(I_{a_{\gamma_{2}}} \cup I_{b_{\gamma_{2}}}\right) \cap\left(I_{a_{\gamma_{1}}} \cup I_{b_{\gamma_{1}}}\right)=\emptyset .
\end{aligned}
$$

Therefore, $J_{\gamma_{1}} \subset \operatorname{Int}_{I} J_{\gamma_{2}}$. Similarly, $K_{\gamma_{1}} \subset \operatorname{Int}_{I} K_{\gamma_{2}}$. This proves Claim d.

Claim e. $I-\{p\}=\left(\bigcup_{\gamma \in \Gamma} J_{\gamma}\right) \cup\left(\bigcup_{\gamma \in \Gamma} K_{\gamma}\right)$ and $\bigcup_{\gamma \in \Gamma} J_{\gamma}$ and $\bigcup_{\gamma \in \Gamma} K_{\gamma}$ are mutually separated.

Proof. By Claim a, $p \in \operatorname{Int}_{I}\left(L_{\gamma}\right)$ for all $\gamma \in A$ so $p \notin J_{\gamma}$ and $p \notin K_{\gamma}$ for all $\gamma \in A$. Suppose $\bigcup_{\gamma \in \Gamma} J_{\gamma}$ and $\bigcup_{\gamma \in \Gamma} K_{\gamma}$ are not mutually separated and $q$ is a point of $\bigcup_{\gamma \in \Gamma} J_{\gamma}$ which is a limit point of $\bigcup_{\gamma \in \Gamma} K_{\gamma}$. Then $q \in J_{\alpha}$ for some $\alpha \in \Gamma$. There exists $\beta \in \Gamma$ such that $a_{\alpha}<a_{\beta}<\pi_{1}(p)$. So $J_{\alpha} \subset \operatorname{Int}_{I} J_{\beta}$ and $J_{\beta} \cap \bigcup_{\gamma \in \Gamma} K_{\gamma}=\emptyset$. So $q$ is not a limit point of $\bigcup_{\gamma \in \Gamma} K_{\gamma}$. Similarly, $\bigcup_{\gamma \in \Gamma} K_{\gamma}$ does not contain any limit points of $\bigcup_{\gamma \in \Gamma} J_{\gamma}$.

Suppose $x \in I-\{p\}-I_{r} \cup I_{s}$ but $x \notin\left(\bigcup_{\gamma \in \Gamma} J_{\gamma}\right) \cup\left(\bigcup_{\gamma \in \Gamma} K_{\gamma}\right)$. By the induction hypothesis, $I_{\pi_{1}(p)}$ is totally disconnected. So whether or not $\pi_{1}(x)=\pi_{1}(p)$, there exist $\gamma \in \Gamma$ and $a_{\gamma}$ and $b_{\gamma}$ so that $x \notin L_{\gamma}$. So then $x \in J_{\gamma} \cup K_{\gamma}$. Therefore, $I-\{p\}=\left(\bigcup_{\gamma \in \Gamma} J_{\gamma}\right) \cup\left(\bigcup_{\gamma \in \Gamma} K_{\gamma}\right)$. This proves Claim e.

Therefore every point $p$ of $I$ with $\pi(p) \notin\{r, s\}$ is a cut point of $I$. So $I$ must contain a Hausdorff arc. This establishes Lemma 2.4. 
THEOREM 2. If $X$ is a Hausdorff arc and $X$ is not first countable at any of its points and $n$ is a positive integer, then every nondegenerate subcontinuum of $X^{n}$ contains an arc.

PROOF. We shall now prove the theorem by induction. Clearly the theorem is true if $n=1$. Suppose then that the theorem is true for all integers less than $n$. Suppose that $\hat{M}$ is a nondegenerate subcontinuum of $X^{n}$ which contains no arc. By Lemma 2.4 there exists a subcontinuum $M$ of $\hat{M}$, an integer $k$, and elements $r$ and $s$ in $X$ so that the set of components of $M \cap\left(X^{k-1} \times[r, s] \times X^{n-k}\right)$ that intersect both $M \cap\left(X^{k-1} \times\{r\} \times X^{n-k}\right)$ and $M \cap\left(X^{k-1} \times\{s\} \times X^{n-k}\right)$ is infinite. Without loss of generality we can assume that $k=1$. Also, if $r \leq \hat{r}<\hat{s} \leq s$ then the set of components of $M \cap\left([\hat{r}, \hat{s}] \times X^{n-1}\right)$ that intersect both $\{\hat{r}\} \times X^{n-1}$ and $\{\hat{s}\} \times X^{n-1}$ is infinite. Thus, let us assume that $[r, s]$ has been chosen so that $r$ is a type II point. Let $C_{1}, C_{2}, \ldots$ be an infinite subsequence of components of $\left([r, s] \times X^{n-1}\right) \cap M$ that intersect both $\left(\{r\} \times X^{n-1}\right) \cap M$ and $\left(\{s\} \times X^{n-1}\right) \cap M$. Let $x_{i} \in C_{i} \cap\left(\{r\} \times X^{n-1}\right)$. Then some subsequence $\left\{x_{n_{i}}\right\}_{i=1}^{\infty}$ has a sequential limit point $x$. So $\left\{C_{n_{i}}\right\}_{i=1}^{\infty}$ has a limiting set $C$ and $C$ must be a continuum. Also $C \cap\left(\{s\} \times X^{n-1}\right) \cap M \neq \emptyset$ and $C \cap\left(\{r\} \times X^{n-1}\right) \cap M \neq \emptyset$, and $C$ is a subcontinuum of $M$. Also, we may assume that $C_{n_{i}} \cap C=\emptyset$ for all $i$ since $C \cap C_{j} \neq \emptyset$ for at most one integer $j$. Let $x_{i}=\left(x_{i}^{1}, x_{i}^{2}, \ldots, x_{i}^{n}\right)$ so that $x_{i}^{1}=r$ for all positive integers $i$. Further, there is an integer $2 \leq l \leq n$ so that the set $\left\{x_{i}^{l}\right\}_{i=1}^{\infty}$ is infinite. Let us suppose that $\left\{x_{i}\right\}_{i=1}^{\infty}$ has been chosen so that $x_{i}^{l}=x_{j}^{l}$ if and only if $i=j$. Let $\left\{O_{i}\right\}_{i=1}^{\infty}$ be a sequence of basic open sets so that $O_{i}=\prod_{m=1}^{n}\left(u_{i}^{m}, v_{i}^{m}\right), x_{i} \in O_{i}$, $\bar{O}_{i} \cap C=\emptyset$, and $\left[u_{i}^{l}, v_{i}^{l}\right] \cap\left[u_{j}^{l}, v_{j}^{l}\right]=\emptyset$ for all $i \neq j$. Thus $\bar{O}_{i} \cap \bar{O}_{j}=\emptyset$ for all $i \neq j$. Let $a_{i}^{l}, b_{i}^{l}$ be such that $u_{i}^{l}<a_{i}^{l}<x_{i}^{l}<b_{i}^{l}<v_{i}^{l}$. Let $R_{i}$ be the basic open set

$$
R_{i}=\prod_{m=1}^{l-1}\left(u_{i}^{m}, v_{i}^{m}\right) \times\left(a_{i}^{l}, b_{i}^{l}\right) \times \prod_{m=l+1}^{n}\left(u_{i}^{m}, v_{i}^{m}\right)
$$

and $x_{i} \in R_{i}$. Let $L_{i}$ be the component of $C_{i} \cap \bar{R}_{i}$ containing $x_{i}$, so $L_{i} \subset([r, s] \times$ $\left.X^{n-1}\right) \cap M$ and $L_{i} \cap \operatorname{Bd}\left(\bar{R}_{i}\right) \neq \emptyset$. By the induction hypothesis, every subcontinuum of $M \cap\left(\{r\} \times X^{n-1}\right)$ is degenerate. So $L_{i} \cap\left(\{r\} \times X^{n-1}\right)$ must be totally disconnected, and $\pi_{1}\left(L_{i}\right)$ is nondegenerate because if $\pi_{1}\left(L_{1}\right)$ were degenerate then $\pi_{1}\left(L_{1}\right)$ would be homeomorphic to a nondegenerate subcontinuum of $X^{n-1}$ and hence would contain an arc. Let $L$ be the limiting set of $\left\{L_{i}\right\}_{i=1}^{\infty}$, thus $L \subset C$. Let $K$ be the limiting set of $\left\{\pi_{l}\left(L_{i}\right)\right\}_{i=1}^{\infty}$, then $\pi_{l}(L) \subset K$. For each $i$, $\pi_{l}\left(L_{i}\right) \subset\left[a_{i}^{l}, b_{i}^{l}\right] \subset\left(u_{i}^{l}, v_{i}^{l}\right)$ and $\pi_{l}\left(L_{j}\right) \cap\left(u_{i}^{l}, v_{i}^{l}\right)=\emptyset$ whenever $i \neq j$, so no point of $\pi_{l}\left(L_{i}\right)$ lies in $K$. So by Lemma 2.2, every point of $K$ is a type I point; but $\pi_{l}(L) \subset K$, so every point of $\pi_{l}(L)$ is a type I point; but $\pi_{l}(L)$ is connected, so by Lemma 2.3, $\pi_{l}(L)$ is degenerate.

For each $i$ the set $\pi_{i}\left(L_{i}\right)$ is nondegenerate and $r \in \pi_{1}\left(L_{i}\right)$, so there exists $q_{i}>r$ so that $[r, q] \subset \pi_{1}\left(L_{i}\right)$. But $r$ is a type II point so no subsequence of $\left\{q_{i}\right\}_{i=1}^{\infty}$ converges to $r$ and hence there is a point $q$ such that $r<q<q_{i}$ for all $i$. So $[r, q] \subset \pi_{1}\left(L_{i}\right)$ for all $i$, so $\pi_{1}(L)$ is nondegenerate. Thus

$$
L \subset \prod_{i=1}^{l-1} X \times\left\{\pi_{l}(L)\right\} \times \prod_{i=l+1}^{n} X .
$$


Thus $L$ is a nondegenerate continuum which embeds in $X^{n-1}$. But by the induction hypothesis $L$ contains an arc, this is a contradiction. So the theorem is established.

Since no hereditarily indecomposable continuum contains an arc, we have

COROLlaRY. If $X$ is a Hausdorff arc and $X$ is not first countable at any of its points then, for each positive integer $n, X^{n}$ does not contain a hereditarily indecomposable continuum.

REMARK. Thus Bing's theorem [B] that every two-dimensional metric continuum contains a hereditarily indecomposable continuum does not generalize to Hausdorff continua.

\section{REFERENCES}

[B] R. H. Bing, Higher dimensional hereditarily indecomposable continua, Trans. Amer. Math. Soc. 71 (1951), 267-273.

[GT] J. Grispolakis and E. P. Tymchatyn, Semi-confluent mappings and acyclicity, Houston J. Math. 4 (1978), 343-357.

[M] R. L. Moore, Foundations of point set theory, Amer. Math. Soc. Colloq. Publ., vol. 13, Amer. Math. Soc., Providence, R. I., 1962.

[S] M. Smith, The irreducibility of continua which are the inverse limit of a collection of Hausdorff arcs, Fund. Math. 94 (1977), 65-73.

Department of Mathematics, Auburn University, Auburn, Alabama 36849 